\title{
Correlation of Serum Bile Acids with Liver Enzymes and Perinatal and Maternal Outcome in Cholestasis in Pregnancy
}

\author{
Satinder Kaur, Anju Huria, Jasbinder Kaur', Pradip Kumar \\ Dept. of Obs /Gyn \& Department of Biochemistry' \\ Govt. Medical College and Hospital, Chandigarh.
}

\begin{abstract}
Aim: To evaluate the correlation of serum bile acid levels with usual liver function tests in patients with obstetric cholestasis and the perinatal outcome of these patients.

Method: It is a prospective analysis of 32 women diagnosed with obstetric cholestasis. Liver function tests and serum bile acid levels were measured at the time of diagnosis and then every two weekly to see the trends.

Result: There was statistically significant increasing trend in the mean values of liver enzymes when followed antenatal till labor and followed by drastic fall in postpartum. The incidence of spontaneous vs. induced labor of preterm and term was $21.8 \%$ vs. $25 \%$ and $21.8 \%$ vs. $31.2 \%$ respectively. $71.9 \%$ patients delivered vaginally while $28.1 \%$ underwent cesarean section. The incidence of low birth weight, birth asphyxia, meconium aspiration syndrome and respiratory distress was $62.5 \%, 15.6 \%, 6.3 \%$ and $28.1 \%$ respectively

Conclusion: Serum aminotransferases are equally or even more sensitive marker of cholestasis to predict perinatal outcome when compared to serum bile acids. Thus in the routine practice, diagnosis and management of obstetric cholestasis can be done on the basis of serum aminotransferases in places where laboratory measurement of serum bile acids are not freely available.
\end{abstract}

Key words: Cholestasis, pregnancy, bile acids, liver enzyme, perinatal outcome

\section{Introduction}

Cholestasis of pregnancy also referred to as obstetric cholestasis, remains widely disregarded as an important clinical issue, though it is extremely stressful to the mother and the baby. It is a disorder unique to pregnancy characterized by maternal pruritus, mild jaundice and liver function impairment presenting mainly in third trimester and recurring in subsequent pregnancies. ${ }^{1,2}$ The incidence of the disease varies considerably between geographic areas ranging from $4 \%$ of normal pregnancies in Chile, $1-1.5 \%$ in Spain to $0.6 \%-0.7 \%$ in U.K. ${ }^{3}$ The frequency among pregnant women in Indian and Pakistani Asians is $1.24 \%{ }^{4}$ The exact pathogenesis of obstetric cholestasis is not known although multiple hypothesis like high estrogen levels in pregnancy, defect in secretion of sulfated progesterone and genetic mutations in multidrug resistant (MDR3) or ABCB4 genes have been proposed. ${ }^{5-7}$

Biochemically cholestasis is characterized by moderate increase in serum aminotransferases (2-10 times of normal) in approximately $99 \%$ of patients and serum bilirubin in $22-56 \%$ of cases. ${ }^{8,9}$ Serum alkaline phosphates levels in these patients is much higher than in the healthy pregnant females. A couple of recent studies have also reported varying levels of gamma glutamyl transpeptidase from normal to elevated in 
$39-50 \%$ of obstetric cholestasis. ${ }^{9,10}$ Although routine liver function tests are widely used, serum bile acids levels are still considered to be the most sensitive and specific indicators of the disease. ${ }^{11}$ There is a marked elevation in the level of serum bile acids (10-100 fold). ${ }^{8}$ Various studies have suggested the role of serum bile acids levels in the surveillance of perinatal outcome in pregnancy with cholestasis but as yet it has not been established whether absolute values of these correlate with adverse pregnancy outcomes or not. ${ }^{8}$

Though the symptoms of intrahepatic cholestasis of pregnancy are severe and incapacitating, there are few, if any sequel to mother. ${ }^{12}$ Various studies have reported an increased chance of caesarean section rate and post-partum hemorrhage, but overall prognosis of the mother is excellent. ${ }^{[13]}$ Perinatal complications associated are increased perinatal mortality rate, preterm delivery, abnormal intrapartum fetal heart pattern, meconium stained liqor, intrauterine growth retardation and fetal distress $.8,14$

Till date optimal management of this disease is still under debate. Active management in the form of close antepartum fetal monitoring and delivery at thirty seven weeks helps to decrease the rate of still births. Various drugs such as cholestyramine, S-adenosylL- methionine, dexamethasone and ursodeoxycholic acid have been used to reduce the intensity of maternal pruritus and improve fetal outcome, however no specific treatment modality has been found to be effective. $^{1}$

The maternal serum bile acid levels have emerged as a significant marker for the diagnosis of obstetric cholestasis, but they are not routinely available and the liver enzymes (serum aminotransferases and total serum bilirubin) are usually relied upon for diagnosis and management of intrahepatic cholestasis of pregnancy in many countries. The present study was undertaken to evaluate the serum bile acid levels in these patients and their values were correlated with usual liver function tests. We further studied the maternal and perinatal outcome and their correlation with serum bile acids.

\section{Methods}

This was a prospective study carried out in our institution in which a total of 32 patients were recruited. Healthy pregnant females more than 28 weeks period of gestation, with singleton pregnancy, having generalized pruritus and raised liver enzymes were enrolled in the study after taking proper consent. Patients having pruritus with rash, history of gallstones, clinical and viral markers suggestive of viral hepatitis, abnormal hepatobiliary system on ultrasound, gestational hypertension and preeclampsia were excluded. Liver function tests \{total serum bilirubin (TSB), alanine aminotransferases (ALT), aspartate aminotransferases (AST), alkaline phosphates (ALP), gamma glutamyl transpeptidase (GGT) $\}$ and serum bile acid levels were measured at the time of diagnosis and then every two weekly to see the trends. Total bile acid was considered to be pathologically raised when concentration exceeded $14 \mu \mathrm{mol} / 1 .{ }^{15}$ Normal value of both ALT and AST was considered to be 0-35 IU/L.

The patient was monitored with fetal movement records, biophysical profile as frequently as clinically indicated and the pregnancy was terminated at 37 weeks or earlier if at any time fetal jeopardy was detected, or as soon as diagnosed, if more than 37 weeks. Liver function tests as well as the bile acids were again measured at the time of labor. Strict intrapartum fetal monitoring was done using continuous cardiotocography. At the time of delivery, period of gestation, duration of the labor, mode of delivery and approximate blood loss was assessed. APGAR scores at 1 and 5 minutes and birth weights were recorded in all neonates. Detailed examination of all babies was done by the pediatrician to rule out any congenital malformation, respiratory distress syndrome and meconium aspiration syndrome.

The women were then observed in their postpartum period for any complications. These patients were followed up after two weeks and their liver function tests and total bile acid levels were repeated.

Statistical analysis: The statistical analysis was performed using Mann-Whitney test (Wilcoxon rank sum test) through SPSS software.

\section{Results}

Over a period of one and a half years from Jan 2006 to June 2007 a total of 38 patients were enrolled in the study. Of these 32 completed the study, five were lost to follow up and one patient progressed to acute fatty liver of pregnancy during the study and was thus excluded. The mean age group in our study was $25.46 \pm 3.04$ years. Sixty two percent of the subjects were primipara and $34.4 \%$ and $3.1 \%$ were para second and third respectively. The mean gestation age was $34.6 \pm 2.40$ weeks and $36.5 \pm 7.34$ weeks at diagnosis and at delivery respectively. In patients diagnosed with cholestasis after 37 weeks pregnancy was terminated as soon as diagnosed hence range of period of gestation at delivery was on higher side.

There was statistically significant increasing trend in the mean values of liver enzymes (AST, ALT, GGT and serum bile acid) when followed antenatal till labor. This was followed by drastic fall at 15 days postpartum in all of these liver enzymes. The mean values of liver 
Table 1. Liver function tests in patients during antenatal, labor and postpartum period.

\begin{tabular}{lllll}
\hline Liver function test & $\begin{array}{l}\text { MEAN } \pm \text { S.D. } \\
\text { Antenatal } \\
\text { (Day 0) } \\
\mathbf{n}=\mathbf{3 2}\end{array}$ & $\begin{array}{l}\text { Antenatal } \\
\text { (Day 15) } \\
\mathbf{n}=\mathbf{1 4}\end{array}$ & $\mathbf{\text { Labor }}$ & $\begin{array}{l}\text { Post partum } \\
\text { (Day 15) } \\
\mathbf{n}=\mathbf{3 2}\end{array}$ \\
\hline S.Bilirubin (Total)m $\mathrm{g} / \mathrm{dl}$ & $0.94 \pm 0.49$ & $0.79 \pm 0.18$ & $1.10 \pm 0.60$ & $0.41 \pm 0.22$ \\
ASTIU/L & $101.75 \pm 73.33$ & $103.35 \pm 50.86$ & $129.37 \pm 83.46$ & $19.06 \pm 5.66$ \\
ALTIU/L & $122.62 \pm 89.20$ & $126.57 \pm 84.75$ & $146.06 \pm 99.26$ & $21.53 \pm 6.24$ \\
Alkaline Phosphatase IU/L & $536.81 \pm 273.45$ & $534.78 \pm 344.26$ & $568.12 \pm 90.87$ & $49.21 \pm 53.85$ \\
Gamma Glutamyl & & & & \\
Transpeptidase IU/L & $36.53 \pm 17.34$ & $40.07 \pm 18.40$ & $44.71 \pm 18.03$ & $18.31 \pm 7.90$ \\
Bile Acid (Total) $\mu \mathrm{mol} / \mathrm{L}$ & $24.58 \pm 19.06$ & $29.50 \pm 16.63$ & $39.03 \pm 21.81$ & $6.30 \pm 4.45$ \\
\hline
\end{tabular}

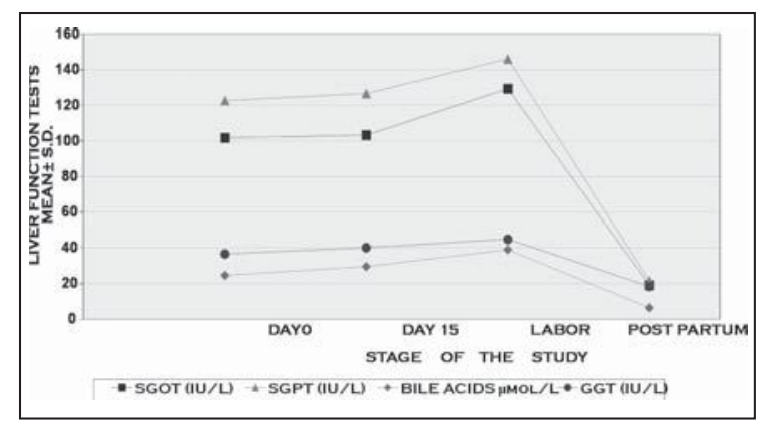

Fig 1. Trend of LFT $s$ in patients at various stages of study.

function tests and their trends are shown in Table 1 and Fig I.

The correlation of serum bile acid levels with liver function tests at the time of diagnosis is shown in Table 2. The mean AST, ALT, ALP values of the patients with raised serum bile acid at the time of diagnosis (antenatal day 0) were significantly higher than the patients with normal serum bile acids levels. Thus, serum aminotransferases are as good a sensitive marker as serum bile acids for the diagnosis of obstetric cholestasis. When these values were further compared at the time of labor, the mean AST, ALT of the patients with raised serum bile acid were higher than those with normal serum bile acids levels. The relationship was not statistically significant, which could be because of the fact that sample size of our study was small (Table 3).

On studying the perinatal outcome of our patients, the total incidence of prematurity was $46.8 \%$ which is much higher than incidence of preterm delivery in normal patients $(8 \%)$. The incidence of spontaneous vs. induced labor of preterm and term $21.8 \%$ vs. $25 \%$ and $21.8 \%$ vs. $31.2 \%$ respectively. This suggests that, obstetric cholestasis leads to an increased rate of induced as well as spontaneous preterm labor.
The majority of patients delivered vaginally $(71.9 \%)$ as compared to $28.1 \%$ who underwent cesarean section. Major indication of cesarean section was fetal distress (abnormal CTG). Postpartum hemorrhage was seen in $18.7 \%$ patients.

The mean values of the liver function test of our patients at labor were then compared with the maternal outcome to see for any correlation. Mean values of total serum bile acid and AST were 43.60 $\pm 23.16 \mu \mathrm{mol} / \mathrm{L}$, $137.66 \pm 95.27 \mathrm{IU} / \mathrm{L}$ in preterm as compared for term deliveries in which they were $34.99 \pm 20.39 \mu \mathrm{mol} / \mathrm{L}$, $122.05 \pm 73.71 \mathrm{IU} / \mathrm{L}$. Though the values were higher in preterm, they were not statistically significant $(\mathrm{p}=$ $0.19,0.86)$. The mean value of ALT $(132.06 \pm 92.17$ IU/L) in preterm was lower than in term patients $(158.41 \pm 106.41 \mathrm{IU} / \mathrm{L})$ the difference being statistically insignificant $(\mathrm{p}=0.25)$.

The incidence of low birth weight, birth asphyxia, meconium aspiration syndrome and respiratory distress was $62.5 \%, 15.6 \%, 6.3 \%$ and $28.1 \%$ respectively. No congenital malformation, still birth and neonatal death was observed. Mean values of liver enzymes in patients with neonatal complications were higher than those without complications, but the difference again was not statistically significant.

\section{Discussion}

Diagnosis of obstetric cholestasis is made on clinical grounds, laboratory tests are essential to reinforce it and to rule out other diseases. The most commonly elevated liver function tests are total bile acids and aminotransferases. Majority of the investigators consider serum bile acid measurement as the gold standard for the diagnosis of disease. Shaw et al evidently regarded elevated levels of serum bile acids as sine quo non for the diagnosis of obstetric cholestasis. ${ }^{16}$

In our study of all pregnant patients with pruritus the diagnosis was established on basis of serum 
aminotransferases. We found that at the time of diagnosis $71.9 \%$ of patients had raised serum bile acids. These patients were followed up and there serum aminotransferases compared with serum bile acid levels which have been claimed to be diagnostic for obstetric cholestasis. On analyzing our results we found that both serum aminotransferases and bile acids increase through the course of pregnancy, and when plotted on a graph there is a parallel increase in the trends from the time of diagnosis till labor.

Then we compared the mean values of aminotransferases in our patients having normal vs. raised serum bile acids $(>14 \mu \mathrm{mol} / \mathrm{L})$, at the time of diagnosis, to see for any statistical correlation. The mean AST and ALT level of the patients with raised serum bile acids were higher than the patients with normal serum bile acids levels, the difference being statistically significant ( $p=0.046$ and 0.049 respectively).

Laatikainen et al in their prospective Scandinavian study in 1977 found no longitudinal correlation between the cholic acid (the most commonly elevated individual bile acids in their series) and AST or ALT. ${ }^{17}$ they concluded that serum concentration of total bile acids and aminotransferases do not change in parallel with either each other or clinical symptoms in obstetrical cholestasis. On extensive review of literature we could not find any study (except the above one) in which the correlation has been studied between the liver function tests and serum bile acids. On the basis of above discussion we conclude that although serum bile acids are considered as gold standard in the diagnosis of obstetric cholestasis, AST and ALT are reliable alternative markers to be used in the routine practice. However, further studies with larger sample size are required to substantiate our data.

Although not life-threatening, the pruritus affects maternal comfort and sense of well-being and is associated with an increased incidence of preterm labor, cesarean section and postpartum hemorrhage. ${ }^{13}$ The results of our study support the increase in rate of these complications. On correlating the mean levels of liver enzymes with maternal outcome, it was seen that the patients with increased prematurity and cesarean section had a higher level of these enzymes. In contrast, the patients with post-partum hemorrhage had lower mean values of these enzymes. This difference between the group with and without complications had no statistical significant relationship. This helps us to conclude that there may be no correlation between maternal complications and levels of any of the liver enzymes.

The course of obstetric cholestasis in the fetus is however not benign, as this disease has been related to number of neonatal complications. ${ }^{[8,14]}$ Though the underlying cause of perinatal and fetal morbidity and mortality remains unclear, it is due to either because of retained bile salts in the placental compartment leading to adverse effects on the placental circulation or on uterine contractility. ${ }^{8}$ In our study although perinatal morbidity was higher than in general population, no mortality was reported as we had followed the policy of active management, with strict antenatal and intrapartum monitoring and termination of pregnancy at 37 weeks. Thus the results of current study demonstrate that with active intervention of such pregnancies the perinatal outcome improves in comparison with previous reports.

We also tried to correlate the above neonatal complications with the mean level of serum bile acids, AST and ALT. Though the mean values of liver enzymes studied are increasing in parallel with neonatal complications, no statistically significant correlation between the degrees of abnormality of liver function tests with perinatal outcome was seen. This could be because of the small sample size of our study or the relationship might turn out to be significant only at markedly deranged levels.

The findings of the Heikkinen ${ }^{18}$ et al, Fisk ${ }^{19}$ et al and Singh ${ }^{20}$ et al support our observations of no significant correlation between neonatal complication rate and liver function tests. However few authors (Laatikainen ${ }^{17}$ et al, Glantz $\mathrm{A}^{14}$ and Bacq et $\mathrm{al}^{10}$ have observed that very high levels of serum bile acids could be related to the above neonatal complications.

We further did a detailed analysis of our data and we could find nine patients at the time of diagnosis of cholestasis who inspite of having raised serum aminotransferases had normal serum bile acids. On following up these patients, serum bile acids became abnormal (>14 $\mu \mathrm{mol} / \mathrm{L})$ in six of these, whereas three continued to have normal levels. The perinatal outcome in these three patients was also analyzed. One of these was induced while two went into spontaneous labor. All of these delivered vaginally, one had post-partum hemorrhage, two had preterm deliveries and small for gestation age babies each and one baby suffered respiratory distress due to prematurity. This observation thus, suggests that normalcy of bile acids levels in women with cholestasis doesn't rule out the risk of perinatal morbidities. The raised serum aminotransferases in the above group with perinatal complications can help us to conclude that serum aminotransferases are equally or even more sensitive marker of cholestasis and to predict perinatal outcome.

Hence in the routine practice, diagnosis and management of obstetric cholestasis can be done on 
the basis of serum aminotransferases in places where laboratory measurement of serum bile acids are not freely available.

\section{Conclusion}

From the above discussion we may conclude that there is a parallel increase in the trends of serum aminotransferases and bile acids from the time of diagnosis till labor as well as there is a significant relationship between the serum aminotransferases and bile acids at diagnosis. Hence, we can use either of these biochemical parameters for the diagnosis and follow up of obstetric cholestasis in routine practice.

In our study although perinatal morbidity was higher than in general population, no mortality was reported as we had followed the policy of active management, with strict antenatal and intrapartum monitoring and termination of pregnancy at 37 weeks.

There is no statistically significant correlation between the degrees of abnormality of liver function tests with perinatal outcome in spite of rising trends. This could be because of the small sample size of our study or the relationship might turn out to be significant only at markedly deranged levels. Hence, large prospective multicentric trials need to be done to detect a biochemical parameter which may correlate with maternal and neonatal outcome.

\section{Acknowledgements}

We acknowledge the support of all teaching and technical staff of Department of Obstetrics and Gynecology and Biochemistry at Govt Medical College and Hospital' Sector 32, Chandigarh.

\section{References}

1. Williamson C, Hems LM, Goulis DG, Walker I, Chambers J, Donaldson O, et al. Clinical outcome in a series of obstetric cholestasis identified via patient support group. Br J Obstet Gynaecol. 2004; 111:676-81.

2. Hay JE. Liver disease in pregnancy. Hepatology. 2008; 47:1067-76.

3. Kenyon AP, Piercy CN, Girling J, Williamson C, Tribe RM, Shennan AH. Pruritus may precede abnormal liver function tests in pregnant women with obstetric cholestasis: a longitudinal analysis. Br J Obstet Gynaecol. 2001; 108:1190-2.

4. Abedin P, Weaver JB, Egginton E. Intrahepatic cholestasis of pregnancy: prevalence and ethnic distribution. Ethn Health. 1999; 4:35-7.

5. Hardikar W, Kansal S, Ronald PJ, Oude Elferink, Peter Angus. Intrahepatic cholestasis of pregnancy: When should you look further? World J Gastroenterol. 2009; 15 :1126-9.

6. Reyes H, Sjovall J. Bile acids and progesterone metabolites in intrahepatic cholestasis of pregnancy. Ann Med. 2000; 32:94-106.

7. $\mathrm{Y}$ Bacq, C Gendrot, F Perrotin, L Lefrou, S Chretien, V Vie-Buret, et al. ABCB4 gene mutations and single-nucleotide polymorphisms in women with intrahepatic cholestasis of pregnancy. J Med Genet. 2009; 46:711-5.

8. Anna PK, Girling JC. Obstetric cholestasis. Progress in obstetrics and gynecology, Vol 16. John Studd, editor. NewYork: Churchill livingstone; 2005.

9. Brites D, Rodrigues CM, Von ZH, Brito A, Silva R. Relevance of bile serum acid profile in the diagnosis of intrahepatic cholestasis of pregnancy in a high incidence area: Portugal. Eur J Obstet Gynaecol Reprod Biol. 1998; 80: 31-8.

10. Bacq Y, Sapey T, Brechot MC, Pierre F, Fignon A, Dubios F. Intrahepatic cholestasis of pregnancy: A French prospective study. Hepatology. 1997; 26:358-64.

11. Heikkinen J, Maentausta O, Ylostalo P, Janne O. Changes in serum bile acid concentration during normal pregnancy in patient with intrahepatic cholestasis of pregnancy and in pregnant women with itching. Br J Obstet Gynaecol. 1981; 88:240-5.

12. Gupta A, Kakkar T, Gupta Y, Hak J. Cholestasis of Pregnancy. J of Obstet Gynaecol India. 2009; 59:320-3.

13. Ray A, Tata RJ, Balsara R, Singhal S. Cholestasis of Pregnancy. J of Obstet Gynaecol India. 2005; 55:247-50

14. Glantz A, Marschall HU, Mattsson LA. Intrahepatic cholestasis of pregnancy: Relationships between bile acid levels and fetal complication rates. Hepatology. 2004; 40:46774.

15. Qureshi MY, Smith SM, Murphy GM. Colorimetric enzymatic measurement of serum total 3- hydroxyl bile acid without extraction. J Clin Pathol. 1984; 37:317-20.

16. Shaw D, Frohlich J, Wittmann BA, Willms M. A prospective study of 18 patients with cholestasis of pregnancy. Am J Obstet Gynecol.1982; 142:621-5.

17. Laatikainen T, Ikonen E. Serum bile acids in cholestasis of pregnancy. Obstet Gynecol. 1977; 50:313-8.

18. Heikkinen J, Maentausta O, Tuimala R, Ylostalo $\mathrm{P}$,janne O. Amniotic fluid bile acids in normal and pathologic pregnancy. Obstet Gynecol.1980; 56:60-4.

19. Fisk NM. Storey GN. Fetal outcome in obstetric cholestasis. Br J Obstet Gynaecol.1988; 95:113743.

20. Singh G, Sidhu K. Cholestasis of pregnancy: A prospective study. Med J of Armed Forces of India. 2008; 64:343-5. 